\title{
EDITORIAL
}

\section{Reimplanting Previously Infected Device in the Same Patient: A Clever Way to Provide Essential Therapy}

\author{
THOMAS CRAWFORD, M.D. and KIM A. EAGLE, M.D. \\ From the Cardiovascular Center, University of Michigan Health System, Ann Arbor, Michigan
}

Since the introduction of pacing, and subsequently defibrillation, cardiac rhythm management devices (CRMDs) have brought the improvement in quality of life as well as extended longevity of patients with sick sinus syndrome, complete heart block, and life-threatening ventricular arrhythmias. Unfortunately, despite decades of widespread use, the benefits of CRMDs do not reach all patients who need them. There remains a great disparity in access to this essential therapy between patients in the advanced economies and low- and middle-income countries, largely due to high cost. ${ }^{1}$ In order to improve access to pacemakers and defibrillators in underserved nations, organizations such as My Heart Your Heart, Pace4Life, and many others have partnered with the funeral and crematory industry to harvest CRDMs postmortem for their potential reuse. ${ }^{2}$ Funeral home director surveys and patient/general population surveys indicate support for this concept. $^{3}$

Over the years, many single center and mostly uncontrolled and retrospective studies have suggested that pacemaker reuse may be safe and effective, offering patients a therapeutic option they are otherwise denied due to lack of insurance and/or resources. ${ }^{4}$ Recent publications have added to the body of evidence that CRMDs may be safely reimplanted. Kantharia et al. ${ }^{5}$ have reported on a retrospective cohort of 53 patients who received postmortem refurbished pacemakers in Mumbai, India. No infections or device-related failures occurred over mean followup of 21 months. Ninety-five percent of patients reported marked improvement in their symptoms. In another report, Pavri et al. ${ }^{6}$ described a consecutive series of 81 patients who underwent implantation of 106 implantable cardioverter defibrillators obtained either postmortem or during device upgrades. No infectious complications occurred during a mean follow-up of 825 days,

Address for reprints: Thomas Crawford, M.D., Cardiovascular Center, The University of Michigan Health System, 1500 East Medical Center Drive SPC 5853, Ann Arbor, MI 40109-5853. Fax: 734-936-7026; e-mail: thomcraw@med.umich.edu

Received April 16, 2014; accepted June 8, 2014.

doi: $10.1111 /$ pace. 12457 and there was no malfunction related to the reused device. Appropriate therapy (shocks or antitachycardia pacing) was delivered in $54.3 \%$ patients, undoubtedly saving many lives. Neither of the two studies had a control arm.

Nava et al. ${ }^{7}$ added to our knowledge of CRMD reuse when they presented data on 603 consecutive patients in an ambispective noninferiority study. The study group patients $(\mathrm{n}=307)$ received resterilized pacemakers, $96 \%$ of them from cadaveric donation, and the control group patients $(\mathrm{n}=296)$ received a new pacemaker. A combined end point of three major outcomes-unexpected battery depletion, infection, and device malfunction-was reached in $5.5 \%$ in the control group and $7.2 \%$ in the study group $(\mathrm{P}=0.794)$. Five new pacemakers $(1.7 \%)$ and 11 resterilized pacemakers $(3.6 \%)$ had unexpected battery depletion $(\mathrm{P}=0.116) ; 3.7 \%$ new pacemakers and $3.2 \%$ reused pacemakers had a procedure-related infection $(\mathrm{P}=0.466)$; and one pacemaker in the study group malfunctioned. The authors concluded that other than the expected shorter battery life, reuse of pacemaker generators was not inferior to the use of new devices.

In this issue of Pacing and Clinical Electrophysiology, Feng and colleagues report on 212 patients who were treated for CRMD infection at the Peking University People's Hospital, Beijing, China. ${ }^{8}$ All patients underwent a removal of their CRMD. One hundred thirteen patients could afford and underwent reimplantation of a brand new CRMD (control group). Ninety-nine patients underwent reimplantation of the infected device after cleaning and resterilization (study group). Following extraction, CRMDs were placed in a solution of $70 \%$ ethanol for 30 minutes, then washed with pipe cleaners and other instruments to make sure that all debris was removed from the orifices of the devices. CRMDs had to have at least 4 years of expected longevity remaining. CRMDs were packed and sterilized with 24-hour ethylene oxide gas. All patients in the study group underwent reimplantation of the same pacemaker generator.

The primary end point in the study was a composite of infection, unexpected battery depletion, or device malfunction, and was reached in 10 patients-five in the study group and 
five in the control group. Recurrent infection occurred in three patients in the study group and two patients in the control group $3.0 \%$ vs $1.7 \%$; relative risk, $0.96 ; 95 \%$ confidence interval, 0.35-2.03; $\mathrm{P}=0.561$ ). There was no premature battery depletion in either group. Five patients experienced device malfunction-high pacing threshold and/or failure to sense-two of who were in the study group and three in the control group. As in the prior studies, brand new leads were implanted with refurbished devices. The cost of the lead(s) usually represents less than $10-15 \%$ of the cost of the device, and either the families or charities have provided the necessary funds to cover this expense.

This study is a valuable addition to the growing body of evidence supporting CRMD reuse. It offers a pragmatic approach for patients with limited resources who develop CRMD infection. Device extraction is necessary in order to erad-

\section{References}

1. Mond HG, Proclemer A. The 11th world survey of cardiac pacing and implantable cardioverter defibrillators: Calendar year 2009A World Society of Arrhythmia project. Pacing Clin Electrophysiol 2011; 34:1013-1027.

2. Baman TS, Crawford T, Sovitch P, Meier P, Sovitch N, Gakenheimer L, Kirkpatrick J, et al. Feasibility of postmortem device acquisition for potential reuse in underserved nations. Heart Rhythm 2012; 9:211214.

3. Gakenheimer L, Lange DC, Romero J, Kirkpatrick JN, Sovitch P, Oral $\mathrm{H}$, Eagle KA, et al. Societal views of pacemaker reutilization for those with untreated symptomatic bradycardia in underserved nations. J Interv Card Electrophysiol 2011; 30:261-266.

4. Baman TS, Meier P, Romero J, Gakenheimer L, Kirkpatrick JN, Sovitch P, Oral H, et al. Safety of pacemaker reuse: A metaanalysis with implications for underserved nations. Circ Arrhythm Electrophysiol 2011; 4:318-323.

5. Kantharia BK, Patel SS, Kulkarni G, Shah AN, Lokhandwala Y, Mascarenhas E, Mascarenhas DA. Reuse of explanted permanent icate device-related bacteremia or device pocket infection. ${ }^{9}$ Patients with already limited resources face a double challenge if their device becomes infected during the immediate postoperative period. The study also opens the door to the possibility of harvesting infected CRMDs in the high-income countries in order to offer them to patients in lowand middle-income countries, after appropriate cleaning and resterilization. While the study was not randomized-the assignment into the treatment group was made based on the patient's ability to fund a new implant-it shows that reimplantation of CRMD is safe and effective. Further studies are needed before the practice of pacemaker reuse can be embraced. A prospective multicenter trial, based on a validated sterilization and electrical testing protocol, is needed to provide the definitive data on safety and efficacy. Should such study succeed, it will be impossible to ignore its results, and innumerable lives will be saved.

pacemakers donated by funeral homes. Am J Cardiol 2012; 109:238240.

6. Pavri BB, Lokhandwala Y, Kulkarni GV, Shah M, Kantharia BK, Mascarenhas DA. Reuse of explanted, resterilized implantable cardioverter defibrillators: A cohort study. Ann Intern Med 2012; 157:542-548.

7. Nava S, Morales JL, Marquez MF, Barrera F, Gomez J, Colin L, Brugada J, et al. Reuse of pacemakers: Comparison of short and longterm performance. Circulation 2013; 127:1177-1183.

8. Feng Z, Xuebin L, Ping Z, Haicheng Z, Long W, Ding L, Jiangbo D, et al. Reuse of infected cardiac rhythm management devices in the same patients: A single-center experience. Pacing Clin Electrophysiol 2014; 37:940-946.

9. Baddour LM, Epstein AE, Erickson CC, Knight BP, Levison ME, Lockhart PB, Masoudi FA, et al. Update on cardiovascular implantable electronic device infections and their management: A scientific statement from the American Heart Association. Circulation 2010; 121:458-477. 